Stefano Fabbiani ORCID: 0000-0002-8720-7228 Asistente Departamento de Farmacología y Terapéutica. Residente Medicina Intensiva.

Federico Garafoni ORCID: 0000-0002-2629-3273 Asistente Departamento de Farmacología y Terapéutica. Residente Medicina Intensiva.

Valentina Catenaccio ORCID: 0000-0003-4236-610X

Asistente Departamento de Farmacología y Terapéutica. Pediatra.

Noelia Speranza ORCID: 0000-0002-3330-9974

Profesor Agregado Departamento de Farmacología y Terapéutica. Farmacóloga. Pediatra

\title{
Eficacia y seguridad de los AINE tópicos
}

\author{
Efficacy and safety of topical NSAIDs \\ Eficácia e segurança dos AINEs tópicos
}

Resumen: Introducción: los antiinflamatorios no esteroideos (AINE) constituyen uno de los grupos farmacológicos más prescritos a nivel mundial. En los últimos años ha aumentado el uso de AINE de administración tópica, con una prevalencia de prescripción creciente. Objetivo: revisar la eficacia y seguridad de AINE tópicos comparados con los administrados por vía oral en el tratamiento del dolor. Material y método: se realizó una revisión narrativa utilizando la base de datos Pubmed. La búsqueda arrojó un total de 141 artículos de los cuales 4 cumplían con los criterios de inclusión propuestos. Discusión: la evidencia que respalda el uso de AINE tópicos en cuanto a eficacia está comparada con placebo, incluyen escaso número de pacientes y utilizan variables primarias blandas, lo que constituye una limitante metodológica para determinar su beneficio, por lo que no se pudo analizar la evidencia disponible según el objetivo planteado. En cuanto a la seguridad, la incidencia de efectos adversos más frecuentemente reportados son los gastrointestinales, siendo menos frecuente con la administración tópica, sin embargo, ésta vía agrega efectos adversos cutáneos en el sitio de administración. Conclusión: la facilidad de acceso a estos medicamentos, la percepción de menor riesgo por tratarse de una vía no sistémica y la escasa evidencia disponible favorecen el uso irracional de los AINE tópicos.

Palabras clave: eficacia, seguridad, AINE, vía tópica, crema, gel, parche, dolor.

Abstract: Nonsteroidal anti-inflammatory drugs (NSAID) are one of the most prescribed pharmacological groups worldwide. In recent years the use of topical NSAID has increased, with a growing prevalence of prescription. The aim of the present work was to review the efficacy and safety of topical NSAID compared to oral administration for the treatment of pain. A narrative review was made using the Pubmed database. The evidence that supports the use of topical NSAID in terms of efficacy is compared with placebo, include a small number of patients and use soft primary outcomes, which constitutes a methodological limitation to asses their benefit, not allowing to analyze the available evidence according to the aim set. Regarding safety, the incidence of adverse effects most frequently reported are gastrointestinal, which are less frequent with topical administration, however, this route adds adverse cutaneous effects at the administration site. The ease of access to these medications, the low risk perception due to it is a non-systemic route and the limited available evidence stimulate the irrational use of topical NSAID.

Key words: efficacy, safety, NSAID, topic route, topic administration, cream, gel, patch, pain.

Resumo: Os medicamentos anti-inflamatórios não esteroides (AINE) são um dos grupos farmacológicos mais prescritos em todo o mundo. Nos últimos anos, o uso de AINE tópicos aumentou, com uma crescente prevalência de prescrição. O objetivo do presente trabalho foi revisar a eficácia e segurança dos AINE tópicos em comparação com a via oral no tratamento da dor. Uma revisão narrativa foi feita usando o banco de dados Pubmed. A evidência que suporta o uso de AINE tópicos em termos de eficácia é comparada com placebo, inclui um pequeno número de pacientes e usa variáveis primárias moles, o que constitui uma limitação metodológica para determinar seu benefício, portanto, a evidência disponível não pôde ser analisada de acordo com o objetivo. Em relação à segurança, a incidência de efeitos adversos mais frequentemente relatados é gastrointestinal, sendo menos freqüente com a administração tópica, porém, esta via acrescenta efeitos cutâneos adversos no local de administração. A 
facilidade de acesso a esses medicamentos, a percepção de menor risco, por ser uma via não sistêmica e a limitada evidência disponível, favorecem o uso irracional de AINE tópicos.

Palavras-chave: eficácia, segurança, AINE, vía tópica, creme, gel, patch, dor.

Recibido: 22/08/2019 Aceptado: 07/10/2019

Departamento de Farmacología y Terapéutica. Facultad de Medicina. Universidad de la República (UdelaR). Uruguay. Correspondencia: E-mail: noeliasperanza@gmail.com 


\section{Introducción}

Los antiinflamatorios no esteroideos (AINE) constituyen uno de los grupos farmacológicos más prescritos a nivel mundial, siendo un grupo heterogéneo de compuestos que presentan actividad analgésica, antiinflamatoria y antipirética. Entre sus múltiples indicaciones, se utilizan para el tratamiento de diversos procesos dolorosos, inflamatorios y febriles, habitualmente como tratamiento sintomático. Su bajo costo, sumado a las condiciones heterogéneas de venta existentes en nuestro medio, que admiten la publicidad directa en algunos casos y son de venta libre (decreto 18/989) ${ }^{(1)}$ hace que el autoconsumo sea muy elevado y la percepción de riesgo que se tiene de estos fármacos sea muy baja.

La vía dérmica admite el uso de cremas, ungüentos, geles, pomadas y parches, permitiendo el pasaje de principios activos a través de las capas de la piel hasta la circulación sistémica. Ésta depende de la concentración del principio activo y el tiempo de exposición al mismo. De modo que, aquellas formas farmacéuticas que aseguren mayor tiempo de exposición y mayor concentración de fármaco, por ejemplo parches, facilitarán un efecto sistémico. Por lo tanto, la vía tópica no es exclusiva para el tratamiento de afecciones locorregionales. Tal es el caso, por ejemplo, de la aplicación de cremas con estrógenos para los síntomas del síndrome climatérico $(2-8)$.

El uso tópico evita el primer paso hepático y las fluctuaciones de las concentraciones plasmáticas, prolonga la duración de acción y mejora la adherencia al tratamiento, reduce además la exposición sistémica de forma significativa comparado con la vía oral, mientras que produce concentraciones clínicamente efectivas en el tejido objetivo ${ }^{(2-8)}$. Como limitantes se destaca que las presentaciones farmacéuticas administradas por esta vía necesitan cumplir con determinadas características como lipofilia, bajo peso molecular y vehículos (excipientes) adecuados. En cuanto a las pautas de administración no se dispone de evidencia para su dosificación. En general, lo que se encuentra recomendado en las fichas técnicas de los AINE es la aplicación de tres a cuatro veces al día para geles y pomadas, y dos veces al día en caso de parches, dada la cinética de absorción lenta de la vía tópica ${ }^{(2-8)}$. Por ello, para la prescripción deben considerarse los siguientes determinantes: la concentración del principio activo, el grosor de la capa aplicada y la forma de aplicación, sea esta como vendaje o fricción. Otro aspecto de relevancia clínica es el vehículo, que altera la velocidad de absorción y la penetrancia del preparado. Éstos pueden ser sólidos, semisólidos o líquidos. La mayoría de los vehículos presentan además acciones sobre la piel; éstas pueden variar desde acciones oclusivas previniendo la pérdida de hidratación por la evaporación, hasta la extracción de lípidos de la capa córnea para reducir su función de barrera.

Por último es de importancia considerar la variabilidad inter e intraindividual, la región anatómica, la edad, las condiciones ambientales (hidratación de la superficie cutánea) y la presencia de otras patologías concomitantes ${ }^{(2-8)}$.

Es probable que el masaje con la aplicación tópica contribuya al efecto terapéutico por aumento del flujo sanguíneo local, en adición al efecto psicológico de la aplicación en el sitio de la noxa ${ }^{(9)}$.

Los AINE de aplicación tópica disponibles en Uruguay, según lo publicado en la página de internet del Ministerio de Salud Pública a la fecha son: diclofenac en aerosol $1 \%$, gel $1 \%$ y $2 \%$, crema $1 \%$, etofenamato gel $5 \%$, ibuprofeno en crema y gel $5 \%$, ketoprofeno crema $2,5 \%$, ketoprofeno parches $30 \mathrm{mg}$ y piroxicam gel $0,50 \%{ }^{(10)}$.

Dado que en nuestro medio no están disponibles las indicaciones aprobadas para cada uno de estos fármacos, se tomó como referencia las de la Agencia Española de Medicamentos y Productos Sanitarios (AEMPS) ${ }^{(11-15):}$

Diclofenac: alivio local del dolor y la inflamación leves y ocasionales producidos por pequeñas contusiones, golpes, distensiones, tortícolis u otras contracturas, lumbalgias y esguinces leves producidos como consecuencia de una torcedura.

Etofenamato: alivio local del dolor y de la inflamación leve y ocasionales producidos por pequeñas contusiones, golpes, distensiones, tortícolis u otras contracturas, lumbalgias y esguinces leves producidos como consecuencia de una torcedura.

Ketoprofeno: afecciones dolorosas, flogísticas o traumáticas de las articulaciones, tendones, ligamentos y músculos (artritis, periartritis, artrosinovitis, tendinitis, tenosinovitis, bursitis, contusiones, distorsiones, luxaciones, lesiones meniscales de la rodilla, tortícolis, lumbalgias). 
Ibuprofeno: alivio local del dolor y de la inflamación leves y ocasionales en adultos y adolescentes mayores de 12 años, producidos por pequeñas contusiones, golpes, distensiones, tortícolis $u$ otras contracturas, lumbalgias y esguinces leves producidos como consecuencia de una torcedura.

Piroxicam: adultos y niños mayores de 12 años para el alivio local del dolor y de la inflamación leve y ocasional

A pesar de la vía de administración, es posible observar efectos sistémicos. Los AINE por vía tópica producen una concentración plasmática máxima que llega a 15\% de la alcanzada por la administración oral de una dosis similar. Esto determina que los efectos adversos dosis dependiente serían menos frecuentes pero pueden observarse ${ }^{(16)}$

Por vía tópica, los efectos adversos más frecuentes son a nivel local, donde se puede observar erupción cutánea, urticaria, descamación y dermatitis de contacto ${ }^{(17-19)}$. Está descrito un caso de hepatitis aguda no fatal por diclofenac tópico, en una paciente portadora de una cirrosis biliar ${ }^{(20)}$.

En los últimos años ha aumentado el uso de AINE de administración tópica, con una prevalencia de prescripción creciente, conformando un $6 \%$ del mercado de Estados Unidos en la última década, aún combinados con la vía oral, basado en un criterio de selección empírico, con escasa evidencia que lo sustente ${ }^{(5)}$. Se desconoce si, en algunas situaciones, éstos son capaces de sustituir o retrasar el uso de los AINE sistémicos.

Existen pocos estudios que evalúen el consumo de este grupo de AINE. En un estudio observacional de utilización de AINE tópicos de Zaragoza realizado durante el año 2010 se observó que 4,92\% ( $n=843)$ del total de pacientes que se atienden en el centro de salud analizado se le prescribieron AINE tópicos. El grupo etario con más prescripciones fue el de 65 a 79 años, y la mayoría eran mujeres (64\%). Los fármacos tópicos más prescritos fueron diclofenaco $(27,33 \%)$, dexketoprofeno (26,67\%) y etofenamato (20,67\%). A 36,7\% de la muestra se le indicó un AINE oral concomitantemente. No se observaron reacciones adversas ${ }^{(21)}$.

En nuestro medio no se cuenta con datos de consumo de AINE.

El objetivo del presente trabajo fue revisar la eficacia y seguridad de AINE tópicos comparados con los utilizados por vía oral en el tratamiento del dolor.

\section{Material y Métodos}

Se realizó una revisión narrativa utilizando la base de datos Pubmed. Se realizaron dos búsquedas, en la primera se utilizaron como palabras clave: «Anti-Inflammatory Agents, NonSteroidal» AND «Administration, Oral» AND «Administration, Cutaneous» AND «Treatment Outcome» y en la segunda se utilizaron como palabras clave: "Administration, Cutaneous» AND «Anti-Inflammatory Agents, Non-Steroidal».

Se utilizó como límite para la búsqueda los ensayos clínicos controlados (ECC). Se analizaron todos los artículos de los que se accedió al texto completo según la pertinencia del título y resumen en función del objetivo planteado.

De la primer búsqueda se obtuvieron 9 resultados de los cuales se analizó uno; el resto no se analizaron por no ser pertinentes y a uno de ellos no se obtuvo acceso al texto completo. De la segunda búsqueda se hallaron 132 citas, de las que se analizaron 3 por ser adecuados para el objetivo formulado.

\section{Resultados}

Un ensayo clínico comparó la eficacia de ibuprofeno tópico versus oral, mediante la aleatorización de 100 adultos con lesión aguda de tejidos blandos en dos grupos, a uno se le administró ibuprofeno en tabletas de $400 \mathrm{mg}$ y a otro ibuprofeno en gel al 5\%, ambos 3 veces al día por 7 días. La variable primaria fue el tiempo necesario para sentirse "completamente mejor" o "casi completamente mejor". La media de tiempo para alcanzar la variable primaria fue de 14 días para el gel y 13,5 días para la vía oral $(p=0,59)$. Ambos tratamientos fueron bien tolerados y solo se reportaron 6 eventos adversos, que no fueron relacionados al tratamiento. Al final del estudio se interrogó a los participantes si tenían preferencia por alguna de las vías de administración, asumiendo ambas como efectivas: $71 \%$ de los pacientes manifestaron preferencia por el tratamiento tópico ( $70 \%$ en el grupo de gel y $71 \%$ en el grupo de tabletas) y 
sólo $8 \%$ tenía preferencia por la vía oral (10\% de los participantes del grupo gel, $6 \%$ tabletas). El resto de los participantes no expresaron preferencias ${ }^{(22)}$.

Otro trabajo analizó la eficacia y seguridad de diclofenac gel a distintas concentraciones para el tratamiento de esguince de tobillo en pacientes entre 16 y 65 años: diclofenac al 1\% $(n=112)$; mentol al 3\% $(n=77)$ y diclofenac en combinación con mentol a las mismas concentraciones $(n=117)$ versus placebo $(n=75)$. La variable primaria fue el área bajo la curva de intensidad del dolor con una escala de cero a diez a las 24-72 horas post aplicación. No hubo diferencias significativas entre los preparados ni versus placebo. Se observó una mayor incidencia de eventos en el sitio de administración con la presentación combinada ${ }^{(23)}$.

Un tercer ensayo clínico controlado que comparó la eficacia y seguridad de diclofenac gel versus placebo en pacientes con cervicalgia. Se aleatorizaron 72 pacientes adultos, 36 de ellos a diclofenac gel al 1,16\% en 4 aplicaciones diarias por 5 días y otros 36 recibieron placebo. La variable primaria fue dolor al movimiento a las 48 horas de tratamiento. La reducción del dolor fue 3 veces mayor con el gel comparado con placebo $(p<0,0001)$. Sólo se notificó efectos adversos en un paciente, que consistió en cefalea en el grupo placebo, que no fue considerado como relacionado al mismo ${ }^{(24)}$.

La guía de manejo de osteoartritis en adultos del National Institute for Health and Care Excellence (NICE) del año 2008 recomienda ofrecer a los pacientes AINE tópicos o paracetamol vía oral, en adición a la educación del paciente, ejercicio físico y disminución del peso, previo al uso de AINE sistémicos ${ }^{(25)}$.

La guía clínica sobre artritis de mano de la European League Against Rheumatism (EULAR) del 2007 recomienda el tratamiento con AINE tópicos sobre los sistémicos en dolores leves a moderados, ya que estos presentan efectos adversos locales, y a nivel sistémico no los presentan con mayor frecuencia que placebo ${ }^{(26)}$.

\section{Discusión}

Se halló un número muy reducido de ECC para analizar el objetivo planteado.

La evidencia que respalda el uso de AINE tópicos en cuanto a eficacia está comparada con placebo, incluyen escaso número de pacientes y utilizan variables primarias blandas, lo que constituye una limitante metodológica para determinar el beneficio o no de estos fármacos en el tratamiento del dolor agudo.

Dentro de éstas, algunas como las escalas de dolor son más reproducibles, pero no dejan de presentar el sesgo de la subjetividad. A pesar de ello, son las variables que frecuentemente se consideran en ensayos clínicos de dolor.

En cuanto a la seguridad, la incidencia de efectos adversos más frecuentemente reportados son los gastrointestinales, siendo menos frecuente con la administración tópica, sin embargo, ésta vía agrega efectos adversos cutáneos en el sitio de administración.

Los ECC que los comparan con placebo muestran beneficio sobre éste.

La falta de estudios que los comparen con otros tratamientos activos limita la realización de recomendaciones sobre la indicación del uso tópico de AINE, si bien podrían considerarse de utilidad en subpoblaciones de riesgo para el desarrollo de hemorragia gastrointestinal, eventos coronarios e insuficiencia renal, dada la baja tasa de efectos adversos sistémicos alcanzados por esta vía, y para aquellos pacientes con mala adherencia al tratamiento vía oral. Entre ellos se destacan los adultos mayores, polimedicados y aquellos con comorbilidades. Es necesario recordar lo limitante y iatrogénico que resulta para estos grupos el uso indiscriminado de AINE sistémicos.

No hay datos en la literatura consultada sobre cuál vehículo constituye la mejor opción para la elaboración de la forma farmacéutica (geles, pomadas, etc).

Por esta misma razón, tampoco puede establecerse cuál AINE presenta mayor absorción dérmica y efectividad clínica, ni por cuánto tiempo deberían utilizarse, lo que puede tener como consecuencia su uso irracional.

La facilidad de acceso a estos medicamentos, la percepción de menor riesgo por tratarse de una vía no sistémica y la escasa evidencia disponible favorecen el uso irracional de los AINE tópicos. 
Dado su amplio uso, sería importante contar con ECC diseñados adecuadamente para evaluar la eficacia comparativa con la vía oral en el tratamiento del dolor agudo. La aplicación de cremas o parches en la región afectada, tanto por su efecto farmacológico como por su efecto placebo contribuyen al efecto analgésico final y debería considerarse a la hora del diseño de los estudios sobre dolor.

\section{Conclusiones}

La evidencia disponible es escasa y de calidad variable. No es posible responder al objetivo planteado. Los AINE tópicos complementan el tratamiento del dolor, sobre todo en dolores leves, pero el nivel de evidencia en el que se basan estas recomendaciones aún es limitada.

El desarrollo de ECC adecuados podría contribuir al uso racional de analgésicos evitando la exposición sistémica masiva de AINE a la población general y aún más a pacientes con factores de riesgo para la aparición de efectos adversos sistémicos.

\section{Financiación}

La presente investigación no ha recibido ayudas específicas provenientes de agencias del sector público, sector comercial o entidades sin ánimo de lucro.

\section{Conflicto de intereses}

Los autores declaran no tener conflicto de intereses.

\section{Bibliografía}

1- Asociación de Química y Farmacia del Uruguay. Marco regulatorio de los Productos Farmacéuticos y Afines. Montevideo: AQF, 2006.

2- Burkhart C, Morrell D, Goldsmith L. Farmacología dermatológica. En: Brunton LL, Chabner BA, Knollmann BC, ed. Goodman \& Gilman: las bases farmacológicas de la terapéutica. $12^{\mathrm{a}}$ ed; México: McGraw-Hill; 2011.

3- Flórez J. Farmacología dermatológica. En: Farmacología humana. 6ª ed; Elsevier: Madrid, 2014.

4- Pastore MN, Kalia YN, Horstmann M, Roberts MS. Transdermal patches: history, development and pharmacology. Br J Pharmacol. 2015 May; 172(9): 2179-2209.

5- Stanos SP, Tyburski MD. Minor and short-acting analgesics, including opioid combination products. In: Benzon HT, Rathmell JP, Wu CL, Turk DC, Argoff CE, eds. Raj's Practical Management of Pain. 4th ed. Philadelphia, PA: Elsevier; 2008:613-641.

6- Grosser T, Smyth E, FitzGerald GA. . Antiinflamatorios, antipiréticos y analgésicos; farmacoterapia de la gota. En: Brunton LL, Chabner BA, Knollmann BC, ed. Goodman \& Gilman: las bases farmacológicas de la terapéutica. $12^{\mathrm{a}}$ ed; México: McGraw-Hill; 2011.

7- Flórez J. Fármacos analgésicos antipiréticos y antiinflamatorios no esteroideos. Antiartríticos. En: Farmacología humana. 6ª ed; Elsevier: Madrid, 2014.

8- Wiechers JW. The barrier function of the skin in relation to percutaneous absorption of drugs. Pharm Weekbl Sci. 1989;11(6):185-198.

9- Arcury TA, Gesler WM, Cook HL. Meaning in the use of unconventional arthritis therapies. Am J Health Promot. 1999 Sep-Oct;14(1):7-15.

10- Uruguay. Ministerio de Salud Pública. Consulta de medicamentos [bases de datos en internet]. Montevideo: MSP; 2017[ Actualización 2019;acceso: 02/01/2019]. Disponible en: https:// listadomedicamentos.msp.gub.uy/ListadoMedicamentos/servlet/com.listadomedicamentos. listadomedicamentos.

11- España. Agencia Española de Medicamentos y Productos Sanitarios. Ficha técnica. Diclofenaco Kern Pharma 11,6 mg/g gel[Internet]. Madrid: AEMPS, 2011[acceso: 02/01/2019]. Disponible en: https:// www.aemps.gob.es/cima/pdfs/es/ft/74119/74119_ft.pdf

12- España. Agencia Española de Medicamentos y Productos Sanitarios. Ficha técnica. Etofenamato Aspitopic 50 mg/g gel [Internet]. Madrid: AEMPS, 2018 [acceso: 02/01/2019]. Disponible en: https:// www.aemps.gob.es/cima/pdfs/es/ft/56338/56338_ft.pdf 
13- España. Agencia Española de Medicamentos y Productos Sanitarios. Ficha técnica. Ketoprofeno Extraplus 25 mg/g gel [Internet]. Madrid: AEMPS, 2018 [acceso: 02/01/2019]. Disponible en: https:// www.aemps.gob.es/cima/dochtml/ft/58552/FichaTecnica_58552.html

14- España. Agencia Española de Medicamentos y Productos Sanitarios. Ficha técnica. Ibuprofeno Pharmagenus 50 mg/g gel [Internet]. Madrid: AEMPS, 2018 [acceso: 02/01/2019]. Disponible en: https://www.aemps.gob.es/cima/dochtml/ft/63404/FichaTecnica_63404.html

15- España. Agencia Española de Medicamentos y Productos Sanitarios. Ficha técnica. Piroxicam Salvacam $5 \mathrm{mg} / \mathrm{g}$ gel [Internet]. Madrid: AEMPS, 2018 [acceso: 02/01/2019]. Disponible en: https:// www.aemps.gob.es/cima/pdfs/es/ft/58986/FT_58986.pdf

16- Heyneman CA. Lawless-Liday C. Wall GC. Oral versus topical NSAID in rheumatic diseases: a comparison. Drugs. 2000 Sep; 60(3):555-74.

17- Maganji M. Connolly M. Bhatt A. Cutaneous irritancy of an ibuprofen medicated plaster in healthy volunteers Postgrad Med. 2018 Jan;130(1):19-23.

18- Liu DJ, Collaku A, Dosik JS. Skin Irritation and Sensitization Potential of Fixed-Dose Combination of Diclofenac 1\% and Menthol 3\% Topical Gel: Results of Two Phase I Patch Studies. Drug Res 2017; 67: 119-126.

19- Noize P, Bénard-Laribière A, Aulois-Griot M, Moore N, Miremont-Salamé G, Haramburu F. Cutaneous adverse effects of ketoprofen for topical use: clinical patterns and costs. Am J Clin Dermatol. 2010;11(2):131-6.

20- Yerly G, Cereda JM. Severe hepatitis due to percutaneous diclofenac. Gastroenterol Clin Biol. 2008 Oct;32(10):824-5.

21- Yagüe MM, Coscollar I, Muñoz P, López MC, Villaverde MV, Gutiérrez F. Uso de antiinflamatorios tópicos en un centro de salud urbano. Estudio comparativo con la evidencia actual. Med Fam SEMERGEN. 2013; 39(6): 304-308.

22- Whitefield M, O'Kane C, Anderson S. Comparative efficacy of a proprietary topical ibuprofen gel and oral ibuprofen in acute soft tissue injuries: a randomized, double-blind study. J Clin Pharm Ther. 2002 Dec;27(6):409-17.

23- Lai P, Collaku, Reed K. Efficacy and safety of topical diclofenac/menthol gel for ankle sprain: A randomized, double-blind, placebo- and active-controlled trial. J Int Med Res. 2017 Apr; 45(2):647-661.

24- Predel H, Giannetti B, Pabst H, Schaefer A, Hug A, Burnett I. Efficacy and safety of diclofenac diethylamine $1.16 \%$ gel in acute neck pain: a randomized, double-blind, placebo-controlled study. BMC Musculoskelet Disord. 2013 Aug 21;14:250.

25- National Collaborating Centre for Chronic Conditions. Osteoarthritis: national clinical guideline for care and management in adults. London: Royal College of Physicians, 2008.

26- Zhang W, Doherty M, Leeb BF, Alekseeva L, Arden NK, Bijlsma JW, et al. EULAR evidence based recommendations for the management of hand osteoarthritis: Report of a Task Force of the EULAR Standing Committee for International Clinical Studies Including Therapeutics (ESCISIT). Ann Rheum Dis. 2007 Mar;66(3):377-88.

\section{Aporte cada autor al trabajo}

Stefano Fabbiani: concepción y diseño del trabajo, recolección de datos, análisis e interpretación de los datos o resultados, redacción del manuscrito.

Federico Garafoni: concepción y diseño del trabajo, recolección de datos, análisis e interpretación de los datos o resultados, redacción del manuscrito.

Valentina Catenaccio: concepción y diseño del trabajo, recolección de datos, análisis e interpretación de los datos o resultados, redacción del manuscrito.

Noelia Speranza: concepción y diseño del trabajo, recolección de datos, análisis e interpretación de los datos o resultados, redacción del manuscrito, revisión crítica del manuscrito. 